\title{
Transformaciones y nuevas perspectivas profesionales y de la Salud
}

\section{Transformations and new Professional and Academic Perspectives of Health Psychology}

Recibido: Diciembre de 2010.

Revisado: Agosto de 2011

Aprobado: Mayo de 2013

\section{Abstract}

This paper conveys a vision of health psychology in three great moments. The first can be called a professional moment, wherein psychologists are introduced to the health field along with medical professionals, and develop a specific field, thereby trying to relate biomedical aspects to psychological ones. A second, or naïve moment means that health becomes an issue of responsibility of individuals. At academic levels, this coincides with the social construction of health as a dominant approach. Finally, a currently ongoing third stage is health service homogenization or integration. The main issue today is to integrate health policies of most countries through both collective and global action, as per international plans demanded by globalization.

Key words: Health Psychology, psychosocial factors, Professional certification, Globalization, public health.

\section{Adela Garzón Pérez}

Universidad de Valencia, Espańa

Correspondencia. Adela Garzón. Facultad de Psicología. Avda. Blasco Ibáńez, 21, 46010-Valencia. Correo-e: garzon@uv.es. Tel. 34963864552

\section{Resumen}

Se realiza una visión de la Psicología de la Salud en tres grandes momentos. Uno inicial que se puede denominar como profesional, en el que los psicólogos se introducen en el campo de la salud, junto a los profesionales de la medicina y desarrollan un campo específico, intentando relacionar aspectos psicológicos con aspectos biomédicos. Un segundo momento, que denominamos lego, en el sentido de que la salud se convierte en un tema de responsabilidad de las personas. A niveles académicos coincide con la construcción social de la salud como enfoque dominante. Por último, un tercer momento que está en marcha actualmente, es la homogeneización o esquemas de integración de los servicios de salud. Hoy la cuestión central es integrar las políticas sanitarias de la mayoría de los países por medio de actuaciones colectivas y globales; según los planes internacionales exigidos por la globalización.

Palabras clave: Psicología de la Salud, Factores Psicosociales, Certificación Profesional, Globalización, Salud Pública. 
Los trabajos de Crossley, Nicolson y Owens (2001), de Leventhal, Weinman, Leventhal, y Phillips, (2008), así como el de Suls y Rothman (2004) entre muchos otros son tres ejemplos representativos de un replanteamiento actual en la trayectoria de la Psicología de la Salud. Siempre que un área de conocimiento ha recorrido el proceso formal de su institucionalización (definiciones, fechas y hechos fundacionales, áreas de trabajo y líneas de investigación) llega un momento de su historia en el que surgen los replanteamientos. La Psicología de la Salud como disciplina no es una excepción. Sus orígenes se sitúan en los setenta, y pasados cuarenta ańos aparece ya la necesidad de valorar el camino recorrido.

El trabajo de Crossley et al., (2001) aboga por un posicionamiento moderado de los psicólogos de la salud que, en su necesidad de ratificar el modelo biopsicosocial, se han visto obligados a exagerar la medida y cuantificación de la experiencia de la enfermedad y percepción de la salud. Los autores critican la aceptación de la orientación dominante de la Psicología de la Salud por la familiaridad de los profesionales de la medicina con la investigación, la orientación técnica y los protocolos estandarizados. Defiende la necesidad de repensar el camino que está tomando la Psicología de la Salud en cuanto se aleja precisamente de su aportación más específica: la orientación subjetiva y social de las personas hacia los temas de salud y enfermedad.

Leventhal et al., (2008) señalan las limitaciones que tienen para los psicólogos de la salud admitir las presiones derivadas del éxito de la práctica médica basada en la evidencia. Aunque sea aceptable su eficacia, sin embargo no desvela los mecanismos reales que subyacen en los procesos de salud y enfermedad; resaltan la necesidad de planificar intervenciones basadas en modelos teóricos, que integren la práctica basada en la evidencia con el desarrollo de modelos más completos; son éstos los que ayudan a comprender mejor los factores que contribuyen a la salud. Asimismo, Leventhal et al., (2008) intentan combinar una práctica basada en datos estandarizados con otra basada en modelos teóricos para poder ayudar a los individuos a mantener conductas de salud.

Existen más datos reveladores del momento actual de la Psicología de la Salud. La metodología de investigación también avanza y, junto a los modelos tradicionales, se plantean otras estrategias y métodos. Suls y Swain (1993) señalan las ventajas de utilizar el meta-análisis para los investigadores, teóricos y profesionales. Un intento de integrar los datos cuantitativos derivados de diferentes estudios empíricos para superar las limitaciones de la investigación más convencional.

Frente a estos autores y al criterio de Leventhal, muchos profesionales confían en los distintos grupos de investigación, unos voluntarios y otros institucionales, cuyo objetivo central es proporcionar de los profesionales de la salud información, extraída de los resultados obtenidos tanto de la práctica cotidiana como de la colección de registros de ensayos clínicos controlados, para que puedan tomar decisiones "bien documentadas" sobre la idoneidad de una u otra intervención en temas de salud. A modo de ejemplo mencionamos dos centros.

Uno es el centro Cochrane y su base de datos con revisiones sistemáticas. Es un proyecto que nace por la década de 1990 en el que colaboran más de 30.000 profesionales de un centenar de países (para más información sobre esta línea de trabajo, el lector puede acudir a Cochrane Database of Systematic Reviews, 2013). Otro relevante por su doble función profesional y formativa, es el CEBMH de Oxford, fundado por Geddes, dentro de una red nacional de Centros para los Cuidados de Salud Basados en la Evidencia. Tiene una doble finalidad; por un lado facilitar la enseńanza y la práctica profesional de la atención sanitaria basada en la evidencia, haciendo especial hincapié en los temas de salud mental y, por otro lado valorar y difundir los métodos más idóneos para incorporar la investigación en la práctica dentro de la enseñanza y formación de futuros profesionales (Geddes \& Carney, 2009).

Por último, Suls y Rothman realizan una valoración del modelo biopsicosocial adoptado y señalan líneas futuras de actuación para que se pueda establecer una relación más clara entre aspectos biológicos, psicológicos sociales y culturales: la investigación, la formación, la práctica y la implementación de modelos deben estar actualizados. Abogan por una mayor colaboración entre disciplinas, el desarrollo de modelos teóricos y de investigación integradores, y el desarrollo de políticas de formación para los futuros profesionales.

Más contundente es el hecho de que en el siglo XXI están proliferando trabajos planteando el futuro de la Psicología de la Salud en distintos aspectos concretos, pero destacan sobre todo el problema de la formación de los futuros psicólogos de la salud. Por ejemplo, Sheridan (1999) y Keefe 
y Blumenthal (2004) plantean el posible futuro del campo de la Psicología de la Salud y sus profesionales. Estos autores describen e integran una serie de artículos publicados en una sección especial del Health Psychology para conmemorar los 25 años de la aparición de la División 38 de la APA. Hacen una valoración de lo conseguido y resumen los cambios necesarios para conseguir un mayor éxito: cambios en la educación, investigación y práctica; intensificar la evidencia empírica que muestre la eficacia de la intervención de los psicólogos de la salud; la importancia de que este campo se incorpore a la atención primaria de los servicios sanitarios; la necesidad de valorar detenidamente aspectos éticos y legales de la atención telemática en los servicios de salud, abogando por el desarrollo de unas directrices desde la APA y la necesidad de que los psicólogos de la salud "desarrollen y utilicen nuevas habilidades" (pp. 157).

Hoy el campo se encuentra en un proceso auto-reflexivo y se refleja en los trabajos de Rodin y Salovey (1989) en el Annual Review of Psychology, o el de Taylor (1990) en el American Psychologist, o de Wallston (1933). Por último, en el Handbook of Social Problems (Ritzer, 2004), Cockerham señala la urgencia de ver los problemas de salud desde la interacción de estilos y conductas de salud de las personas, factores de la estructura social de un país y sus sistemas de atención médica. Cockerham habla también del binomio agency-structure como un marco teórico de análisis que supera la visión individualista de la salud y establece un juego dinámico entre conductas y estilos de vida en salud y las dimensiones socioestructurales de dichos estilos de vida. En esa misma línea se sitúa el artículo de Phelan, Link y Tehranifar (2010), bajo el título Social Conditions as Fundamental Causes of Health Inequalities: Theory, Evidence, and Policy Implications.

Estos ejemplos, entre otros tantos, muestran que la Psicología de la Salud ha superado ya esa época inicial en el que una nueva área empieza su andadura y se agarra al modelo dominante, en este caso el modelo biológico y médico y el biopsicosocial. Su etapa de crecimiento no solamente está consolidada, sino que además ha logrado incorporarse a la práctica profesional y, en la actualidad, tiene historia y distancia suficiente como para comenzar a realizar autorreflexiones y replanteamientos.

El modelo biopsicosocial de Engels de 1977, que significó la superación del modelo tradicional de medicina del XIX, puede ser replanteado en el siglo XXI. La cuestión está en saber bajo qué orientación y sentido puede ser superado. De momento ya se perfila una corriente bajo el título de Psicología de la Salud Crítica; un exponente es el libro de Radley (1994) en el que se replantea el significado que tiene el concepto de enfermedad. Existe una incipiente crítica a los conceptos claves -salud y enfermedad- como una dimensión continua. Además, una tendencia predominante, en los tiempos actuales es la formulación de planes de actuación que hacen hincapié en aspectos de la salud y la enfermedad, característicos de sociedades de servicios, globalizadas e interdependientes. Planes que tratan de homologar la práctica de todos los profesionales de la salud.

\section{Profesionalización del campo}

Las circunstancias que rodean la aparición de cualquier disciplina siempre se sitúan en distintos planos. Existen factores internos que empujan al nuevo desarrollo, pero éstos suelen ser coherentes con factores ajenos a la ciencia -relativos al desarrollo y necesidades de las sociedades. Los psicólogos de la salud tienen ya un recorrido suficiente como para construir su propia historia. No vamos a describirla -existe una amplia literatura sobre este temapero destacaremos algunos aspectos llamativos que añaden quizá algún elemento a las valoraciones y replanteamientos ya comentados.

Pongamos un primer ejemplo: no deja de ser curioso que se haga especial hincapié en la superación del modelo biomédico, como hecho central para la formación de la Psicología de la Salud, y no se analice lo que sucedió en el propio campo de la psicología a finales de los años sesenta. La Psicología de la Salud surge por razones internas del propio desarrollo de las ciencias, pero no es menos cierto que por esa época la Psicología como ciencia estaba siendo forzada a demostrar la utilidad de sus conocimientos básicos para el bienestar de las sociedades. En los años setenta la Psicología está marcada por su expansión en esferas concretas de la vida social. Aparece inicialmente como una Psicología Aplicada y poco después florecen distintas psicologías aplicadas, en ámbitos tan diversos como la justicia, la política, la educación y, con mucha fuerza, una psicología aplicada a la salud. Es decir, junto al cambio de concepción de la enfermedad dentro de la medicina tradicional, había algo más que no suele comentarse: la propia necesidad de la psicología de estar reconocida y justificada socialmente. Esta motivación es uno de los factores que la encaminó hacia distintas esferas sociales: la salud fue uno de los 
campos iniciales en los que la psicología intentó validar y justificar su existencia.

Junto a la crítica hacia el modelo médico y hacia la excesiva preocupación etiológica predominantes hasta los años setenta, no es menos importante la crítica a una psicología que había demostrado y aportado poco a la solución de los problemas de urgencia social. La Psicología de finales de 1960 se vio acorralada (Seoane, 2005) desde distintos ángulos por su endogamia y falta de atención a los problemas de las personas y las sociedades; se le criticó su inutilidad, su falta de relevancia, su mecanicismo, su confianza ciega en la metodología de la experimentación, el laboratorio, los sesgos estadísticos, y hasta se incluyó entre las críticas su marcado carácter acomodaticio al statu $q u o$-una crítica ideológica del trabajo realizado. Se intentó superar esta situación y se produjo un sinfín de aplicaciones que intentaban demostrar su utilidad indiscutible. Se recurrió a todo tipo de teorías, modelos, viejos resultados experimentales, hipótesis clásicas y resultados de última hora, con tal de que mostrara alguna aplicación a cualquier tipo de problema que tuviera urgencia social y la sociedad se encontró invadida por un repertorio de técnicas psicológicas para la resolución de problemas vitales.

Es en este contexto en el que surge un amplio abanico de estas aplicaciones en el campo específico de la salud: los estudios sobre el impacto del estilo de vida en la salud y las formas de enfermar, sobre todo en las enfermedades crónicas, sobre los modos saludables de vida y recomendaciones para una psicología preventiva de la enfermedad. Tuvieron un gran éxito social los análisis de las reacciones de las personas ante la enfermedad o cualquier crisis de salud, así como el estudio de las atribuciones sobre las causas de su enfermedad. Los problemas psicológicos relacionados con el cáncer son representativos en este sentido (Ibáñez, 2004). Los estudios psicológicos de la enfermedad incluían también la percepción, interpretación y elaboración de los síntomas y de los cambios producidos a lo largo de la crisis. En tercer lugar, se emplearon conceptos psicosociales para explicar la relación entre la persona y el médico o el sistema de salud, tales como el mecanismo de la obediencia o la sumisión ante diversos tratamientos, los efectos de la satisfacción o los procesos de afrontamiento ante la enfermedad.

Con todo, estos factores internos del propio campo de la salud, ya sea por las limitaciones del modelo biomédico, ya sea por las necesidades de la propia psicología, no son suficientes para explicar la expansión, éxito y consolidación de la Psicología de la Salud en la última década del siglo $\mathrm{XX}$. Es necesario acudir a razones externas, relacionadas con factores sociales, económicos y políticos, especialmente las políticas sanitarias de las sociedades desarrolladas. Estos factores externos han sido objeto de análisis y existen descripciones pormenorizadas, tanto en el contexto académico (ej. sociología de la medicina) como en las organizaciones nacionales e internacionales de salud. De estos factores, destacan los relacionados con los cambios culturales, las nuevas formas de entender y afrontar los problemas derivados de la salud y la enfermedad. Para analizar estos cambios culturales, empezaremos por mencionar algunos aspectos sociales que facilitaron la aparición de la Psicología Social de la Salud.

En las sociedades occidentales de servicios, las razones externas de la incorporación de la psicología a los servicios de salud son conocidas. Por un lado, los avances teóricos, técnicos y de investigación médica permiten el control de las enfermedades infecciosas que, junto con otros factores, alargan la vida de las personas y envejecen a las sociedades, tanto que en Ritzer (2004) se toma la longevidad que logran alcanzar las personas como indicador de la calidad de los sistemas sanitarios de un país. Se asiste, en el plano individual, a una nueva forma de estar enfermo y un desplazamiento hacia las enfermedades crónicas (articulares, respiratorias, cardiovasculares, SIDA, etc.). A finales del XIX y primeras décadas del XX, el desarrollo científico de la medicina y su apoyo en otros campos científicos (inmunología, epidemiología, biología, química, etc.) permitieron desarrollar sistemas de control de enfermedades predominantes hasta entonces. A finales de XX, aparecen otras enfermedades y un nuevo desafío: la longevidad de las personas y las sociedades, que hace necesario formular sistemas de afrontamiento de la salud y los sistemas de atención (de las organizaciones nacionales a instancias y directrices internacionales).

Las administraciones nacionales se ven forzadas a aumentar las partidas presupuestarias para la sanidad pública y a desarrollar planes sanitarios que atiendan las necesidades de diagnóstico y tratamiento de las enfermedades, así como la promoción de salud y prevención de la enfermedad (Matarazzo, 1982). La atención primaria pasa a un primer plano y proliferan las directrices de distintas organizaciones, representadas en la OMS y los distintos Institutos Nacionales de Salud, en materia de definición de la salud y clasificación 
de las enfermedades y en el desarrollo de planes estratégicos que permitan desarrollar modelos eficaces de atención sanitaria. Además, antes de 1970, factores posbélicos habían provocado una colaboración de los servicios entre la psicología clínica y la psiquiatría.

Esta colaboración fue ampliándose a través de campos como la medicina conductual y la conducta de salud; después se establece una colaboración más general entre psicología y medicina (Guze, Matarazzo \& Saslow, 1953). Los años 90 van a presenciar un cambio más fuerte en estas alianzas sucesivas entre psicología y medicina, en tanto que nuevos factores sociales favorecen y refuerzan un cambio en la manera de pensar e interpretar los temas de salud; son los cambios culturales que convierten salud y enfermedad en algo más que condiciones biológicas, se convierten también en concepciones sociales (Cockerham, 2004; Taylor, 2010; Twaddle, 2004).

Tres sensibilidades en la Psicología de la salud. La literatura científica actual pone de manifiesto la proliferación de temas y áreas psicológicas que se ocupan de la salud. Más aún, los especialistas de dichas áreas no solo señalan matices relevantes que las distinguen, sino que ofrecen definiciones de cada una de ellas para indicar su particularidad en el estudio de la salud. En el ámbito español, la segunda versión ampliada del texto de Amigo, Fernández y Pérez (2009) hace una revisión rápida de algunas de éstas áreas y sus objetivos diferenciados. Simón (1993) señala los intentos por delimitar las distintas áreas de la psicología aplicada a la salud; entre psicología clínica, psicología comunitaria, medicina conductual, psicología de la salud y algunas otras. En otro ámbito, Ogden $(2002,2007)$, repasa también los distintos orígenes, significado y propósito de las diferentes áreas psicológicas relacionadas con la salud. Es más, son pocos los manuales que no se ven obligados a mencionarlas y a marcar sus diferencias.

Junto a esta diversificación de áreas, o quizá por ello, existe un enorme abanico de cuestiones y temas que tratan actualmente los psicólogos de la salud. En función de la formación profesional dentro de la Psicología (clínico, comunitario, social, educativo, de las organizaciones, etc.), de su actividad (académica o profesional), de sus relaciones con otros profesionales (médicos, sociólogos, antropólogos, enfermeros, cuidadores) y por supuesto de su sensibilidad social, nos encontramos con temas muy dispares que forman en la actualidad el campo de esta área de la psicología. Dicha disparidad va de la fertilidad asistida a los estados terminales, los servicios oncológicos o cardiovasculares a los relacionados con las unidades de dolor, desde los modelos y creencias legas sobre la salud hasta las conductas de salud y experiencia de la enfermedad.

La proliferación de estudios psicosociales sobre los problemas de salud es tan enorme que resulta difícil establecer no solo un listado de aplicaciones concretas en este campo, sino que resulta casi inviable encontrar una categorización de las mismas que sea aceptada por toda la comunidad científica dedicada a la salud. Como vamos a plantear a continuación, quizá la sensibilidad, la ideología, en el sentido amplio de la palabra, y la formación académica de estos profesionales puede ayudar a establecer algún tipo de categorización.

Es evidente que existen muchas formas $-\mathrm{y}$ todas discutibles- de poner en orden y clarificar los temas que ha ido incorporando la Psicología de la Salud a lo largo de sus treinta años de existencia institucionalizada, y podemos partir de dos hechos. Junto a la definición propuesta por Matarazzo (1980) que recoge las líneas principales de actuación mencionadas y cubre los objetivos marcados por los sistemas públicos sanitarios, lo primero que habría que señalar son los objetivos que se marcaron en el nacimiento de la división 38 de la APA, formulados en los términos siguientes: promover las aportaciones de la psicología en la comprensión de la salud y la enfermedad; integrar la información biomédica sobre la salud y la enfermedad con el conocimiento psicológico actual; proporcionar información y aportar datos a la comunidad científica y profesional, así como al público general, de los principales resultados de la investigación, las actividades y servicios; facilitar y promover la formación de profesionales y la creación de servicios especializados en psicología de la salud. (APA, división 38; APA, 1976).

La segunda característica que debe mencionarse es que se ha producido un desplazamiento de la sensibilidad académica hacia una orientación práctica, de servicios sanitarios y de incorporación de los psicólogos a los diferentes niveles de atención sanitaria y sus unidades de intervención.

En función de estos dos criterios una forma de clarificar o, al menos, ordenar la pluralidad de enfoques, teorías, temas y cuestiones específicas que en la actualidad dan forma a la Psicología de la Salud consiste en distinguir como mínimo tres tipos de sensibilidad práctica, que en 
parte son responsables de la falta de integración temática del campo (Seoane, 2005; Seoane y Garzón, 2010).

La primera sería una sensibilidad sanitaria radical, marcada por la preocupación por las enfermedades y por su intento de acoplarse a los profesionales de la medicina. La segunda, la sanitaria existencial prefiere hablar del enfermo, de su experiencia y sus problemas y la tercera, la sanitaria solidaria, afrontan los temas de salud desde la diversidad y particularidades de los grupos humanos y sus formas de enfermar.

Tres ejemplos son representativos de esta sensibilidad sanitaria radical: los textos de Bennet, Perry y Rozensky (2002), el de Broome y Llewelyn (1994) y, en el ámbito español; el texto de Amigo y otros (2003) y el de Simón (1999). Cada uno a su manera integra al psicólogo en el campo de la salud partiendo de la clasificación de las enfermedades y destacando en cada una de ellas los factores psicológicos implicados. En algún caso, incluso parten de las 17 categorías de la Clasificación Internacional de Enfermedades (ICD-9).

La orientación existencial adquiere otros matices en aquellos psicólogos que se centran más en enfermos que en enfermedades, más en las personas y su enfermedad. Analizan los modelos de creencias de salud que están a la base de las cuestiones de diagnóstico y tratamiento; la dinámica de las relaciones entre médico y paciente y sus formas diferentes de interpretar y procesar la información que comparten, así como las implicaciones emocionales y adaptativas de la enfermedad, el tratamiento y la hospitalización. En definitiva, el proceso completo de la experiencia de la enfermedad, desde los síntomas iniciales de malestar, pasando por el proceso del tratamiento hasta la recuperación. Adquieren especial relevancia las teorías relativas a las concepciones legas de la salud y la enfermedad, la percepción de control, el estrés y las estrategias de afrontamiento, junto con las características de personalidad. Al mismo tiempo, tiende a analizar las conductas que facilitan y promueven la salud (ejercicio físico, dieta equilibrada, etc., así como las conductas y hábitos de riesgo (tabaco, alcohol, drogas). Los textos de Marks, Murray, Evans, Willig, Woodall, y Sykes (2008), de Ogden (2002) o de Radley (1994) estarían en esta segunda línea.

Por último, la orientación solidaria es casi una consecuencia de la anterior, puesto que al enfrentarse al enfermo, éste se mueve en distintos grupos de pertenencia que le hacen más o menos vulnerable a determinados estilos y situaciones de vida relacionadas con la salud y a formas diferentes de enfermar y de afrontar individual, social y económicamente dicha situación. Baste como ejemplos más representativos de esta orientación manuales como el Kato y Mann (1996) que abordan desde los problemas de desigualdad social a los problemas específicos de salud en función de edades, sexo, orientación sexual, situación laboral, minorías, etnia, países, etc. o el de Marmot y Wilkinson con el título Social Determinants of Health de 2006. Esta orientación adquiere todavía mayor justificación a partir de la creación en 2005 de la Comisión sobre Determinantes Sociales de la Salud, según la propuesta realizada por el Director General de la Organización Mundial de la Salud celebrada en 2004. Los trabajos ya mencionados de Cockerham y Phelan son otro ejemplo más, entre otros muchos.

A pesar de la diversificación de temas, enfoques y sensibilidades que acabamos de mencionar, también es verdad que existe una sensibilidad más académica que fundamentó los comienzos y desarrollo inicial del nuevo campo - especialmente en las Universidades - y que trataba de aplicar sus conocimientos teóricos de psicología al campo de la salud. En la medida que mantuvo una filosofía global e integradora sobre el papel de la psicología en el campo de la salud y le preocupaba la formación de esos futuros especialistas, esta orientación ha logrado que el campo, diversificado en temas y cuestiones variadas, mantenga sin embargo cierta integración a través de una serie de líneas teóricas y variables destacadas que configuran el panorama actual del trabajo y la investigación más básica. Las revisiones de Rodin y Salovey (1989) y de Salovey, Rothman y Rodin (1998) son quizá las más equilibradas y completas que describen la situación actual (ver también, Salovey y Rothman, 2003).

La salud como responsabilidad del individuo. A finales del siglo XX, la salud deja de ser una cuestión exclusiva de los profesionales de la medicina y otras disciplinas afines, y las personas toman conciencia de la responsabilidad y también del control que tienen sobre su salud. Una concepción coherente con las sociedades de servicios y también en consonancia con las dificultades de los países para mantener los sistemas sanitarios dentro de la responsabilidad del Estado. La salud es un aspecto más de la vida que debemos atender y de la que hay que responsabilizarse junto a los profesionales y los servicios sanitarios; además es necesario poner los medios necesarios 
para no enfermar y, en el caso que ocurra, hay que utilizar los recursos psicológicos y conductuales para facilitar la recuperación; más aún, es necesario hacer una vida saludable, cuidar el cuerpo de forma que esté en plenas facultades: de la preocupación por la enfermedad se pasa a la preocupación por la salud. La moda postmoderna del cuidado personal para mantenerse en forma es un ejemplo de este cambio (Garzón, 2012a; Smith y MacKenzie, 2006) mencionan trabajos pioneros en esta línea.

Aunque en la literatura actual se resaltan más los factores externos relacionados con los avances técnicos, organizativos y sociales que facilitaron el desarrollo de la Psicología de la Salud en comparación con los cambios culturales relacionados con el concepto de salud, existen textos representativos que recogen este desplazamiento de la concepción biológica, centrada en la etiología, el tratamiento y los profesionales de la salud, hacia una construcción social de la salud más orientada al cuidado y la prevención, donde profesionales y legos comparten responsabilidades, por ejemplo Lachmund y Stollberg de 1992 porque, aunque podría verse como un texto de historia de la medicina, en realidad se trata de un conjunto de ensayos sobre la historia social de la medicina desde la perspectiva del construccionismo social y las relaciones entre el médico y el enfermo.

Radley (1993) defiende que el estudio biográfico y la vivencia de la enfermedad son compatibles con la necesidad de estudiar la enfermedad en sus contextos culturales; las personas responden a la enfermedad en función de sus creencias y prácticas diarias aprendidas dentro de sus grupos sociales.

Lupton (1994) en Medicine as Culture: Illness, Disease and the Body in Western Societies hace un repaso de las distintas perspectivas desarrolladas en la sociología médica y plantea abiertamente la crisis cultural de la medicina moderna, por su ineficacia, alto coste, poca regulación y amplia injusticia. Define la enfermedad como un indicador existencial de los seres humanos; al mismo tiempo, plantea la necesidad de comprender las relaciones entre la biología y la cultura para comprender y estudiar la salud.

Mattingly y Garro (2000) presentan la narrativa como método y concepto que permite, a través del análisis de las experiencias de los profesionales de la medicina y las vivencias de los enfermos, poner en relación el plano cultural y el del conocimiento de realidades observables, obteniendo así una visión integral de la salud. El texto surge a raíz de una reunión de la American Antropological Association (publicada en Social Science and Medicine, con el titulo Narrative Representations of Illness and Healing, editado por Garro y Mattingly en 1994). El texto es representativo de una de las perspectivas actuales de la antropología médica. En esta misma dirección de las posibilidades de la narrativa, como método y concepto, habría que mencionar el libro editado por Hurwitz, Greenhalgh y Skultans bajo el título Narrative Research in Health and Illness.

Allsop, Jones y Baggott en 2004 colaboran en el texto editado por Shaw y Kauppinen sobre perspectivas europeas de la construcción social de temas relacionados con la salud y la enfermedad. Los autores destacan sobre todo el análisis de distintos aspectos de la salud desde la perspectiva lega y del consumidor de salud.

A pesar de sus diferencias, todos estos planteamientos comparten algunas características que definen la perspectiva de la construcción social de la salud: 1. Entienden ésta como un valor que trasciende las medidas biofisiológicas del funcionamiento normal de un organismo, 2. La salud es un valor, una realidad deseable, más allá del conocimiento formal, 3. La enfermedad y la salud son experiencias que los individuos adquieren durante sus vidas y que afrontan e interpretan en función de esquemas socialmente compartidos y en los que han sido socializados, 4. Salud y enfermedad son realidades biológicas pero también culturales; se enmarcan en un sistema general de creencias que les proporciona un sentido, un significado personal y colectivo y, además, tienen un carácter histórico y espacial. Son realidades cambiantes por la interpretación social a la que están sometidas, al margen de los cambios producidos por los avances en el conocimiento o la industria médica. En las construcciones sociales de la salud, junto al profesional, la etiología, los tratamientos y la curación, adquiere relevancia el usuario, el conocimiento lego y la autorregulación a través de hábitos y costumbres. En este sentido, cabe mencionar las aplicaciones de la llamada Psicología Positiva, que se describe brevemente en el último apartado, pero que propicia recursos psicológicos relevantes para operacionalizar la idea de la responsabilidad del individuo en el cuidado de su salud.

Esta responsabilidad del individuo en su salud se acompaña académicamente de una amplia gama de posturas 
teóricas desde la perspectiva de la construcción social de la salud; unas son más moderadas y otras más arriesgadas. Las primeras se apuntan a la concepción unidimensional y continua de la salud, frente a la concepción dicotómica del modelo biomédico, tal como señala la definición de la OMS. Otras plantean abiertamente que salud y enfermedad son dos realidades y mundos distintos, cada una con aspectos diferentes y realidades múltiples, pero sobre todo son realidades por las que el individuo pasa una y otra vez a lo largo de su vida. Esta orientación más actual es relevante no solo para los profesionales de las ciencias de la salud (médicos, enfermeras, psicólogos en sus distintas especialidades), sino también para la formación de los futuros profesionales. En cualquier caso es un avance frente a los planteamientos iniciales que surgieron con la aceptación del modelo biopsicosocial de Engel y, desde luego, hace más compleja la historia oficial contada por los psicólogos de la salud (Seoane y Garzón, 2010).

\section{Nuevas orientaciones y agenda en Psicología de la Salud}

Planes globales de actuación. La Psicología de la Salud en el XXI está encontrando, o está obligada, a una nueva fórmula de actuación e integración. Los psicólogos de la salud en su evaluación han ido abarcando nuevas áreas, han desarrollado múltiples enfoques y, al mismo tiempo han proliferado las organizaciones, instituciones y canales de difusión hasta lograr el reconocimiento público y político de su papel en el campo de la salud. Sin embargo, la dispersión y proliferación de enfoques, temas y cuestiones a que dio lugar su etapa de profesionalización se resuelto más por razones externas que por una reflexión interna de la comunidad científica y profesional de psicólogos.

El proceso de globalización, inicialmente económica, ha obligado en las sociedades más desarrolladas a un replanteamiento de los servicios de atención sanitaria que se promueve desde organizaciones internacionales, pero con serios interrogantes sobre si son viables y eficaces tales planes y estrategias de integración (Magtymova, 2007). Las diferencias de países y sus circunstancias, la diversidad de colectivos con necesidades específicas en materia de salud y, al tiempo, la inevitable necesidad de acoplar los gastos sanitarios de los países, en un mundo cada vez más interdependiente y neoliberal en lo económico (Garzón, 2012b; Ward, 2012), han conducido a una época de actuaciones colectivas y globales que tratan de integrar las políticas sanitarias de la mayoría de los países. Son estas unas actuaciones que intentan poner orden y homogeneizar en la esfera pública y de servicios sanitarios, al margen del orden interno de las áreas científicas y campos profesionales implicados.

Dichos planes generales de actuación en política sanitaria se fueron fraguando en la última década del siglo XX y comienzan a plasmar resultados desde el 2000. La Organización Mundial de la Salud (OMS) (2009) señala que "en el siglo XXI, la salud es una responsabilidad compartida, que exige el acceso equitativo a la atención sanitaria y la defensa colectiva frente a amenazas transnacionales". En 2004 se plantea la creación de una Comisión sobre los determinantes Sociales de la Salud, encargada de recoger y analizar datos durante tres ańos (2005-2008) de la mayoría de los países para empezar a racionalizar y distribuir equitativamente la atención sanitaria. En dicho informe analizan y definen conceptos claves para una política mundial de salud más justa. Entre dichos conceptos destacan tres que avalan la necesidad de que el psicólogo de la salud amplíe su visión más allá de los aspectos médicos, clínicos y de clasificaciones psicopatológicas relacionadas con las conductas de enfermar y sanar.

Gradientes sociales: "Los más pobres entre los pobres, en todo el mundo, son también los que tienen peor salud. Dentro de los países, los datos muestran que en general cuanto más baja es la situación socioeconómica de una persona, peor salud tiene. Existe un gradiente social de la salud que discurre desde la cúspide hasta la base del espectro socioeconómico. Se trata de un fenómeno mundial, observable en los países de ingresos altos, medianos y bajos. La existencia de un gradiente social de la salud implica que las inequidades sanitarias afectan a todos" (pp 1-2, Comisión sobre Determinantes Sociales de la Salud, OMS, 2009).

Determinantes sociales: "Se entienden por determinantes sociales de la salud las circunstancias en que las personas nacen, crecen, viven, trabajan y envejecen, y los sistemas establecidos para combatir las enfermedades. A su vez, esas circunstancias están configuradas por un conjunto más amplio de fuerzas: económicas, sociales, normativas y políticas" (pp 1-2, OMS, 2009)

Inequidades sanitarias: "Se entiende por inequidades sanitarias las desigualdades evitables en materia de salud entre grupos de población de un mismo país, o entre países. Esas inequidades son el resultado de desigualdades en el 
seno de las sociedades y entre sociedades. Las condiciones sociales y económicas, y sus efectos en la vida de la población, determinan el riesgo de enfermar y las medidas que se adoptan para evitar que la población enferme, o para tratarla” (pp 1-2, OMS, 2009).

Las actuaciones e informes específicos de la Comisión sobre los determinantes Sociales de la Salud dentro del marco de los Objetivos de Desarrollo del Milenio, establecidos en 2000 en el marco de las Naciones Unidas y presentados como una meta a alcanzar en 2015, están siendo objeto de valoraciones por parte de organismos privados y organizaciones públicas de los distintos países miembros. Sin embargo, este ejemplo es uno entre otras muchas actuaciones y organismos interesados en crear unos Planes Estratégicos de Intervención más o menos globalizadores.

Actualmente las alianzas público-privadas y las denominadas horizontales-verticales tienen especial relevancia y protagonismo como nuevas formas de cooperación internacional en materia de salud. En dichas alianzas intervienen tanto organismos estatales como locales, tanto los profesionales de la salud como organizaciones económicas privadas relacionadas con el sector de salud, y también lo hacen organizaciones no gubernamentales locales y asociaciones de colectivos que impulsan políticas de integración para el intercambio y gestión de conocimientos, instrumentación y profesionales implicados en la actividad sanitaria.

Tratan de homologar los planes e intervención en materia de salud, realizados por equipos formados por personal de la administración, expertos, profesionales, docentes, asociaciones profesionales, un ejemplo, entre muchos, es la unidad de Gestión e Intercambio del conocimiento (Knowledge Management and Sharing, KMS) de la OMS. Del mismo modo, se formulan esquemas homologables internacionalmente en la formación y adquisición de competencias básicas de los futuros profesionales de la salud. Estos tendrán que alejarse de la tradición académica y científica tradicional y acercarse a las realidades sanitarias de cada momento, siguiendo protocolos de actuación dictados por los planes internacionales y atender a las nuevas demandas generadas por las circunstancias específicas de cada país y, al tiempo, por el proceso de globalización, marcado en lo económico por planteamientos neoliberales de mercado y, en lo cultural, por la diversificación de colectivos y enfermos que conviven en las sociedades de hoy, tal como queda reflejado en la Declaración de Objetivos para el nuevo Milenio. En el año 2015 será necesario un replanteamiento a la luz de la crisis actual del modelo político y económico que dominó el siglo anterior.

Tendencias académicas en el nuevo milenio. Una de las consecuencias de los cambios en la visión de la psicología de la salud y de los intentos de integrar las políticas sanitarias ha sido un mayor énfasis - si cabe - en reformular la formación y competencia profesional de los psicólogos de la salud. Desde que la división 38 de la APA intentó una revisión de los planes de estudio de los futuros psicólogos, se han desarrollado diversos enfoques centrados en delimitar cuales son las competencias y habilidades que se requieren para la práctica profesional.

Los modelos formulados, como era de esperar, varían en función de los ámbitos de aplicación (centros de atención primaria, unidades especializadas, centros de salud mental, etc.). Empero, en un intento de resumir los múltiples programas, se podría decir que existen al menos tres dimensiones genéricas que cubren las habilidades básicas para una práctica profesional: una dimensión, clásica, que cubre la formación tradicional (teoría, metodología y técnicas de investigación. Es lo que a veces se denomina competencias formales en el sentido de proporcionar a los futuros psicólogos los conocimientos y aptitudes necesarias para el buen desempeño de su trabajo. Una segunda dimensión recoge el conjunto de habilidades que permite detectar, diagnosticar y programar un plan de actuación ante las necesidades psicológicas y sociales derivadas de un cuadro patológico. En la actualidad tiene más relevancia una tercera dimensión que alude a la necesidad de que los futuros profesionales aprendan a desarrollar las competencias adquiridas en su formación dentro del contexto del trabajo en equipo, junto a otros profesionales que tienen otras funciones y una sensibilidad diferente. Son estas habilidades sociales que deben ser inculcadas desde los primeros años de formación y que les capacita para saber plantear los problemas y sus soluciones desde perspectivas diferentes integrando en su visión profesional (psicología) las perspectivas de los otros profesionales (médicos, psiquiatras, oncólogos, trabajador o asistente social, etc.) con los que tiene que trabajar. En el ámbito espańol basta repasar el listado de competencias formuladas por la Orden Ministerial (BOE, 2013, pág. 44950).

Por ejemplo, France et al. (2008) presentan el Modelo de Competencia aplicado al campo de la psicología 
clínica de la salud, en un intento de identificar las competencias específicas necesarias en la formación de futuros profesionales. Otros han formulado las ventajas de utilizar la experiencia del trabajo cotidiano de los profesionales para formular, desde la investigación basada en la evidencia, modelos generales de formación y entrenamiento. Una apuesta que tiene la ventaja añadida de estrechar y aunar sensibilidades, históricamente dispares, entre la investigación básica y la aplicada, entre el ámbito académico y el profesional. Unas dicotomías que los nuevos planes de integración y globalización tienen que ir erradicando de los planes de estudio y los programas y diseños de intervención (Kazdin, 2008; Kivlighan, 2008; Silverstein y Auerbach, 2009).

Dentro de este contexto de la nueva agenda para la psicología de la salud y sus profesionales, y al margen de los programas de formación y entrenamiento, es necesario incorporar nuevas orientaciones teóricas que están adquiriendo especial relevancia. Nos referimos a la psicología evolucionista (Neuberg, Kenrick \& Schaller, 2010), a los enfoques derivados de la neurociencia con sus nuevos métodos e instrumentos de investigación (Lieberman, 2010) y al enfoque más social y cultural que se podría resumir en las implicaciones para el ámbito de los servicios de salud de la denominada Psicología Positiva (un ejemplo representativo de esta nueva línea es, el trabajo del psicólogo social y de la salud, Wolfgang Stroebe) (Stroebe, 2011, 2013).

Desde que Seligman difundió su formulación de Psicología Positiva, en su conferencia presidencial de la APA en 1998, son muchas las líneas de investigación que se lanzaron para aplicarla al campo de la salud (el lector puede acudir al Positive Psychology Center (PPC) de la Universidad de Pennsylvania para una visión más detallada). Rápidamente se comprendió que frente a una psicología basada en lo patológico y disfuncional se podía utilizar el potencial de emociones y sentimientos positivos de las personas para inculcar la percepción de su fortaleza (empowerment) y, en consecuencia, su capacidad para hacer frente a las dificultades de la vida. Sus implicaciones en el campo de la salud no se hicieron esperar, como tampoco las técnicas para medir ese potencial positivo de las personas. Sin embargo, la idea no es nueva, por los ańos 60 y por razones muy distintas se extendió una corriente en todas las ramas de psicología sobre la capacidad de individuos y colectivos para gestionar y afrontar con sus propias armas internas las dificultades (sociales, políticas, de salud) a las que se enfrentaban. Nos basta con recordar aquí la corriente, por ejemplo, de la Psicología Comunitaria, que por otro lado estuvo estrechamente relacionada con los problemas de salud mental.

Tanto la psicología evolucionista como el enfoque de las neurociencias han sido también incorporadas en el ámbito de los profesionales de la salud. Ambas orientaciones son vistas, en cierto modo, como reacción de un nuevo realismo (biología, realidades físicas, químicas) frente a los excesos de construccionismo social. Esto no quiere decir que sus avances y nuevas líneas de investigación no supongan un progreso en el conocimiento; no cabe duda que permitirán avanzar en lo que resulta tan difícil para la ciencia: cómo explicar esa integración de la realidad biológica, psicológica y social que subyace tanto al bienestar como el no-bienestar de individuos y colectivos. Y más importante, cómo esa integración puede luego avalar los programas de intervención social.

\section{Referencias}

Allsop, J., Jones, K. \& Baggott, R. (2004). Pains, Loss and action collective. Health Consumer Groups and the Policy Process, (pp. 107-123). En I. Shaw \& K. Kauppinen, Constructions of Health and Illness: European Perspectives. Burlington: Ashgate.

Amigo, I., Fernández, C., y Pérez, M. (2009). Manual de Psicología de la Salud. Madrid: Pirámide.

APA Task Force on Health Research (1976). Contributions of Psychology to Health Research: Patterns, Problems, and Potentials, American Psychologist, 31(4), 263-274. doi: 10.1037/0003-066X.31.4.263

APA (División 38) en http://www.health-psych.org/ AboutMission.cfm

Bennet, S., Perry, N.W.Jr., \& Rozensky, R.H. (2002). Handbook ofClinical Health Psychology, vol. 1: Medical Disorders and Behavioral Applications. Washington, D.C: American Psychological Association.

BOE (2013). Orden Reguladora del Máster en Psicología General Sanitaria. BOE no 142, 14-6-13, 449- 450.

Broome, A., Llewelyn, S. (eds.)( 1994). Health Psychology: Process and Applications. London: Chapman and Hall.

Cochrane Database of Systematic Reviews. Issue 6 of 12, June 2013. 
Cockerham, W.C. (2004). Health as a social problem. En G. Ritzer, Handbook of social problem (pp. 281-297). London: Sage.

Crossley, M.L., Nicolson, P., \& Owens, G. (2001). Do we need to rethink health psychology. Psychology, Health and Medicine, 6(3), 243-265. doi: $10.1080 / 13548500123431$.

France, C.R., Masters, K.S., Belar, C.D., Kerns, R.D., Klonoff, E.A., Larkin, K.T., Smith, T.W. \& Suchday, S. (2008). Application of the Competency Model to Clinical Health Psychology. Professional psychology: research and practice, 39(6), 573-580. doi: 10.1037/0735-7028.39.6.573.

Garzón, A. (2012a). Incorporación y adaptación del sistema de creencias postmodernas. Psicothema, 24(3), 442-448.

Garzón, A. (2012b). Tecnificación del conocimiento y creencias sociales. Boletín de Psicología, 106, 29-44.

Geddes JR, \& Carney S. (2009). Evidence-based medicine and neurophysiology. Clinical EEG \& Neuroscience, 40(2), 59-61. doi: 10.1177/155005940904000205

Guze, S.B., Matarazzo, J.D., \& Saslow, G.A. (1953). Formulation of principles of comprehensive medicine with special reference to learning theory. Journal of Clinical Psychology, 9, 127-136. doi: 10.1002/1097-4679(195304)9:2<127::AIDJCLP2270090208>3.0.CO;2-F

Kato, P.M., \& Mann, T. (1996). Handbook of Diversity Issues in Health Psychology. The Plenum Series in Culture and Health. New York: Plenum.

Kazdin, A. (2008). Evidence-based treatment and practice: new opportunities to bridge clinical research and practice, enhance the knowledge base, and improve patient care. American Psychologist, 63(3), 146-59. doi: 10.1037/0003-066X.63.3.146.

Kivlighan, D. M. Jr. (2008). Overcoming our resistances to "doing" evidence-based group practice: a commentary. Journal of Clinical Psychology, 64(11), 1284-1291. doi: $10.1002 /$ jclp.20540

Keefe, F.J., \& Blumenthal, J.A. (2004). Health Psychology: What Will the Future Bring?. Health Psychology, 23(2), 156-157. doi: 10.1037/0278-6133.23.2.156

Hurwitz, B., Greenhalgh, T. \& Skultans, V. (2004). Narrative research in health and illness. New York: Cambridge University Press.
Ibáñez, E. (2004). El papel del psicólogo en los planes nacionales de oncología. Boletin de Psicología, 82, 7-24.

Lachmund, J., \& Stollberg, G. (1992). The Social Construction of Illness: historical, sociological and anthropological perspectives. Coronet Books.

Leventhal, H., Weinman, J., Leventhal, E.A., \& Phillips, L.A. (2008). Health Psychology: The Search for Pathways between Behavior and Health. Annual Review Psychology, 59, 477-505. doi: 10.1146/ annurev.psych.59.103006.093643

Lieberman, M.D. (2010). Social Cognitive Neuroscience. En Susan T. Fiske, Daniel T. Gilbert, \& Gardner Lindzey (eds.) Handbook of Social Psychology (pp. 143-193). US: John Wiley \& Sons, Inc.

Lupton, D. (1994). Medicine as culture: Illness, disease and the body in western societies. London: Sage. Reeditada en 2012.

Magtymova A. (2007). Estrategias para la integración de los servicios de atención de la salud primaria en países de medianos y bajos ingresos en el lugar de la prestación: Comentario de la BSR (última revisión: 25 de septiembre de 2007). La Biblioteca de Salud Reproductiva de la OMS; Ginebra: Organización Mundial de la Salud.

Marks, D.F., Murray, M., Evans, B., Willig, C., Woodall, C. y Sykes, C (2008). Psicología de la Salud. Teoría, Investigación y Práctica. México: Manual Moderno.

Matarazzo, J. D. (1980). Behavioral health and behavioral medicine: Frontiers for a new health psychology. American Psychologist, 1980 35, 807-817.

Matarazzo, J.D. (1982). Behavioral Health's Challenge to Academic, Scientific, and Professional Psychology. American Psychologist, 37 (1), 1-14. doi: 10.1037/0003-066X.37.1.1

Mattingly, C., \& Garro, L.C. (2000). Narrative and the Cultural Construction of Illness and Healing. New York, Cambridge University Press.

Neuberg, S.L., Kenrick D.T. \& Schaller, M. (2010). Evolutionary Social Psychology. En Susan T. Fiske, Daniel T. Gilbert, and Gardner Lindzey (eds.) Handbook of Social Psychology. (pp. 761-796). US: John Wiley \& Sons, Inc.

Ogden, J. (2007). Health Psychology-A textbook. Buckingham: McGraw Hill. 4 ed. 
Ogden, J. (2002). Health and the construction of the individual. London: Routledge.

OMS (2009). Commission on Social Determinants of Health, WHO, 2009. Versión española, Subsanar las desigualdades en una generación. Informe final de la Comisión sobre Determinantes Sociales de la Salud. Argentina (BBAA): Ediciones Journal S.A

Phelan, J.C., Link, B.G., \& Tehranifar, P. (2010). Social Conditions as Fundamental Causes of Health Inequalities: Theory, Evidence, and Policy Implications. Journal of Health and Social Behavior, 51, 28-40. doi: 10.1177/0022146510383498.

Radley, A. (1993). Worlds of Illness: Biographical and Cultural Perspectives on Health and Disease. London: Routledge.

Radley, A. (1994). Making sense of illness. The social psychology of health and disease. London: Sage.

Ritzer, G. (Ed) (2004). Handbook of social problems. London: Sage.

Rodin, J., y Salovey, P. (1989). Health psychology. Annual Review of Psychology, 40, 533-579. doi: 10.1146/ annurev.ps.40.020189.002533.

Salovey, P., \& Rothman, A.J. (eds). (2003). Social Psychology of Health. New York: Psychology Press.

Salovey, P., Rothman, A.J. \& Rodin, J. (1998). Health behavior. The handbook of social psychology (Fourth edition), (pp. 633-683). Boston, Mass: McGraw-Hill.

Seoane, J. (2005). Psicología Social de la Salud. En J. Vallejo, C .Leal (eds), Tratado de Psiquiatría, vol I, (pp. 445-458). Barcelona; Ars Medica.

Seoane, J., y Garzón, A. (2010). Psicología Social de la Salud. En J. Vallejo, C .Leal (eds), Tratado de Psiquiatría, vol. I, (pp. 501-516). Barcelona; Ars Medica.

Sheridan, E.P. (1999). Psychology's Future in Medical Schools and Academic Health Care Centers. Journal of Clinical Psychology in Medical Settings, 6(2), 211-218.

Silverstein, L.B., \& Auerbach, C.F. (2009). Using qualitative research to develop culturally competent evidencebased practice. American Psychologist, 64(4), 274275. doi: $10.1037 / \mathrm{a} 0015439$.
Simón, M.A. (1993). Psicología de la Salud: Aplicaciones clinicas y estrategias de intervención. Madrid: Pirámide.

Simón, M.A. (Ed.)(1999). Manual de Psicología de la Salud. Madrid: Biblioteca Nueva.

Smith, T.W., \& MacKenzie, J. (2006). Personality and risk of physical illness. Annual Review Clinical Psychology, 2, 435-67. doi: 10.1146/annurev. clinpsy.2.022305.095257.

Stroebe, W. (2011). Social Psychology and Health. USA. OPU. McGraw-Hill, 3ed.

Stroebe, W. (2013). Psicología Social de la Salud X (entrevista a Wolfgang Stroebe). http://www.desdeelexilio. com/2013/01/16/psicologia-social-de-la-salud-xentrevista-a-wolfgan-stroebe/

Suls, J., \& Rothman, A. (2004). Evolution of the Biopsychosocial Model: Prospects and Challenges for Health Psychology. Health Psychology, 23(2), 119-125.doi: 10.4236/ojmp.2012.14009

Suls, J., \& Swain, A. (1993). Use of Meta-analysis in Health Psychology. En S. Maes, H. Leventhal, and M. Johnston (eds.). International Review of Health Psychology. Vol 2 (pp. 3-28). Chichester: Wiley.

Taylor, S.E. (1990). Health psychology: The science and the field. American Psychologist, 45, 40-50.

Taylor, S. E. (2010). Health. En Susan T. Fiske, Daniel T. Gilbert, \& Gardner Lindzey (eds.) Handbook of Social Psychology (pp. 698-723). USA: John Wiley $\&$ Sons, Inc.

Twaddle, A. (2004). How medical care systems become social problems. En G. Ritzer, Handbook of social problem (pp. 298-315). London: Sage.

Wallston, K.A. (1993). Health Psychology in the USA. En S. Maes, H. Leventhal, M. Johnston (eds.). International Review of Health Psychology (pp. 215228). Chichester: Wiley.

Ward, S. C. (2012). Neoliberalism and the Global Restructuring of Knowledge and Education. Nueva York: Routledge. 